\title{
Improvement the temperature signal filtering in lock-in thermography
}

\author{
Anna Stoynova ${ }^{1}$, and Borislav Bonev ${ }^{1, *}$ \\ ${ }^{1}$ Tecnical University of Sofia, Department of Microelectronics, 1797 Sofia, Bulgaria
}

\begin{abstract}
At present, lock-in thermography is widely used non-destructive method for defects detection. The informative images in lock-in thermography (e.g. phasegram) are obtained after temperature signal filtering of the raw data. The postprocessing in lock-in thermography is more complex than in other active thermography methods and very important for defects detectability. In some cases the standard postprocessing can significantly decrease the quality of the temperature signal filtering, respectively decreasing defects detectability, although the parameters of lock-in thermography measurement are selected correctly. The aim of the paper is to study the quality of temperature signal filtering in lock-in thermography depending on used offline postprocessing. For this reason, a methodology based on modelling and measurements of temperature signals from infrared thermography for determination of cases, in which the temperature signal filtering quality is decreased significantly, is used and corresponded methods for correction are proposed. The results from modelling and from real lock-in thermography measurements shows that by using of the proposed methods can be avoided decreasing of temperature signal filtering quality due to improper postprocessing. In addition, the proposed methods allow same defect detectability at lower energy, induced in tested sample, which is very useful for materials that are not sufficiently heatresistant.
\end{abstract}

\section{Introduction}

At present, a strong interest exists towards the use of active thermography methods in non-destructive material and structural analyses [1]-[3]. In active thermography, a forced thermal excitation is used. Different kind of excitation sources (optic, electromagnetic, ultrasound etc.) can be employed for intentional temperature change.

The heighten demand especially of lock-in thermography is associated with the both price reduction of the infrared cameras and computing power increase of the used computer systems. This involves complicated postprocessing of thermograms resulting in more accurate quantitative evaluations.

An off-line methodology has been applied for optimization of lock-in frequencies choice in case of heat source modulation into a train of square waves [4]. Complex lock-in analyses have been provided by two alternative low pass filtering methods in the time domain (averaging) and in the frequency domain (harmonics suppression).

In the paper, another off-line methodology is presented to enhance information from lock-in IR signals in case of optical heat source modulation into a train of sin waves. The quality of temperature signal filtering in lock-in thermography depending on used offline postprocessing are studied and analysed. Offered methodology allows using of ordinary IR cameras and low cost experimental setups at the expense of extra time for computational procedures. Improving the quality of the temperature signal filtering also allows improving energy efficiency of the lock-in thermography measurement, as the same phasegram quality can be obtained with lower temperature change [5]. In addition, lower heating energy from the excitator allows testing of objects, which can otherwise be destroyed from the excitation with high heating energy.

The methodology based on modelling and measurements of temperature signals from infrared thermography is used. Some cases, in which the temperature signal filtering quality is decreased significantly, are presented and corresponded methods for correction in such cases are proposed. The proposed methods are merged into a common algorithm for postprocessing in lock-in thermography in order to obtain maximum temperature signal filtering quality. Comparison between results from modelling and real lock-in thermography measurements is presented.

\section{Lock-in thermography processing methods}

In lock-in thermography, periodically illumination to inject thermal waves into the specimen is used. The thermal response is recorded at the same time using infrared camera. The light intensity (when the excitator

\footnotetext{
* Corresponding author: bonev@ecad.tu-sofia.bg
} 
is halogen lamp) may modulate with a sinusoidal waveform signal. The frequency of this signal is named lock-in frequency. The created thermal waves penetrate object's surface and partially reflected by its nonhomogeneities. Due to interference of the reflected portions of the wave with the input thermal wave, phase shifts of the local surface temperature oscillating at the lock-in frequency can be derived. The surface temperature variations are captured via infrared camera in series of thermograms (sequences). The amplitude and the phase of the sinusoidal wave pattern at each pixel after postprocessing are computed. The resultant images are a phasegram and/or an ampligram.

Non-homogeneities of the object are better detected from the phasegrams (and/or ampligrams) than from the raw thermograms because phase images are less affected by surface emissivity variations, uneven heating and environment reflections. Nevertheless, to obtain the temperature change and phase difference, caused only by the excitation source, it is needed temperature signal filtering [4].

One of the methods for temperature signal filtering is digital lock-in correlation [3]. In digital lock-in correlation, two intermediate images are computed - inphase image and quadrature image.

Each specific pixel on in-phase and quadrature images is obtained by [3]:

$$
\begin{gathered}
S^{0^{\circ}(x, y)}=\frac{1}{N n} \sum_{k=1}^{N} \sum_{m=1}^{n} F_{k, m}(x, y) K_{m}^{0^{\circ},} \\
S^{-90^{\circ}}(x, y)=\frac{1}{N n} \sum_{k=1}^{N} \sum_{m=1}^{n} F_{k, m}(x, y) K_{m}^{-90^{\circ}},
\end{gathered}
$$

where $S^{0^{\circ}}(x, y)$ - value of in-phase image's pixel $(\mathrm{x}, \mathrm{y}), S^{90^{\circ}}(x, y)$ - value of quadrature image's pixel $(\mathrm{x}, \mathrm{y})$, $x$ - row of thermogram, $y$ - column of thermogram, $N-$ number of lock-in periods for which the calculation is performed, $n$ - number of captured thermograms per lock-in period, $F_{i, j}(x, y)$ - value of thermogram's pixel $i$ from lock-in period $j, K_{m} 0^{\circ}$ - correlation coefficient for computation of the in-phase image, $K_{m}{ }^{-90^{\circ}}$ - correlation coefficient for computation of the quadrature image.

The correlation functions are [3]:

$$
\begin{gathered}
K_{m}^{0^{\circ}}=2 \sin \left(\frac{2 \pi(m-1)}{n}\right), \\
K_{m}^{-90^{\circ}}=-2 \cos \left(\frac{2 \pi(m-1)}{n}\right),
\end{gathered}
$$
[3]:

Ampligram and phasegram are calculated as follows

$$
\begin{aligned}
& S_{A}(x, y)=\sqrt{S^{0^{\circ}}(x, y)^{2}+S^{-90^{\circ}}(x, y)^{2}}, \\
& S_{P}(x, y)=\tan ^{-1}\left(\frac{S^{-90^{\circ}}(x, y)}{S^{0^{\circ}}(x, y)}\right) .
\end{aligned}
$$

where $S_{A}(x, y)$ - value of the ampligram's pixel $(\mathrm{x}, \mathrm{y})$, $S_{P}(x, y)$ - value of the phasegram's pixel $(\mathrm{x}, \mathrm{y})$.

In [5] is proposed adding of correction coefficients in the correlation function, which can change its frequency and initial phase. In result equations (3) and (4) are transformed as follows:

$$
\begin{aligned}
K_{m}^{0^{\circ}} & =2 \sin \left(\frac{2 \pi(100+d)(m-1)}{100 n}-2 \pi t_{d} f_{l}\right), \\
K_{m}^{-90^{\circ}} & =-2 \cos \left(\frac{2 \pi(100+d)(m-1)}{100 n}-2 \pi t_{d} f_{l}\right),
\end{aligned}
$$

where $t_{d}$ - time shift of the correlation function, $f_{l}-$ lock-in frequency, $\mathrm{d}$ - percentage change in frequency.

\section{Modelling of temperature signal}

The temperature change over time at a given point on the tested object (so called temperature signal) captured with infrared camera in lock-in thermography is shown on Fig. 1.

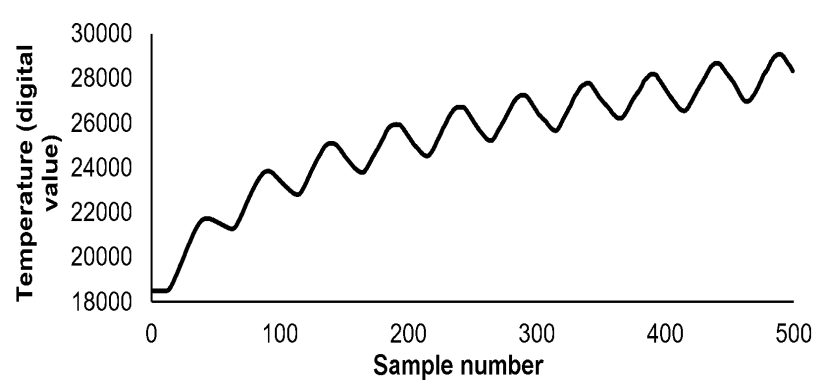

Fig. 1. Temperature signal of a pixel from raw thermogram sequence.

The real temperature signal including noise component and can be modelled with the following equation:

$$
\begin{gathered}
F(k)=F_{\text {mod }}(k)+F_{\text {noise }}(k), \\
F_{\text {mod }}(k)=T_{\text {initial }}+a k+b k^{2}+\Delta T \sin \left(\frac{2 \pi k}{n}+\psi_{F}\right), \\
F_{\text {noise }}(k)=\Delta T_{\text {noise }} \sin \left(\frac{2 \pi k}{n_{\text {noise }}}+\psi_{\text {noise }}\right),
\end{gathered}
$$

where $F(k)$ - model of the temperature signal, $F_{\text {mod }}(k)$ - a component of the model due to thermal modulation, $F_{\text {noise }}(k)$ - a noise component, $T_{\text {initial }}$ - initial temperature, $a$ and $b$ - coefficients, $\Delta T$ - an amplitude of temperature oscillation due to the modulation, $k$ - number between 1 and $n, n$ - number of samples per lock-in period, $\psi_{\mathrm{F}}-$ initial phase of the temperature oscillation, $\Delta T_{\text {noise }}-$ amplitude of temperature oscillation due to the noise, $n_{\text {noise }}-$ number of samples in a noise period, $\psi_{\text {noise }}-$ initial phase of the noise.

The model of phase shifted temperature signals (signal 1 and signal 2) will be used. This model can be 
composed for two different pixels from the infrared sensor array. The phase of each the temperature signal is computed by (6). The parameters values of the model are shown on Table I.

Table 1.The parameters values of the signals model.

\begin{tabular}{|c|c|c|}
\hline Parameter & Value at signal 1 & Value at signal 2 \\
\hline$T_{\text {initial }}\left({ }^{\circ} \mathrm{C}\right)$ & 25 & 30 \\
\hline$a$ & 0.2 & 0.2 \\
\hline$b$ & -0.0002 & -0.000195 \\
\hline$\Delta T\left({ }^{\circ} \mathrm{C}\right)$ & 5 & 4.5 \\
\hline$n$ & 50 & 50 \\
\hline$\psi \mathrm{F}(\mathrm{rad})$ & 0.0628 & 0.0698 \\
\hline$\Delta T_{\text {noise }}\left({ }^{\circ} \mathrm{C}\right)$ & 1 & 1 \\
\hline$n_{\text {noise }}$ & 5 & 8 \\
\hline$\psi_{\text {noise }}$ & 0.0209 & 0.1047 \\
\hline
\end{tabular}

The temperature signals are shown in graphical form in Fig. 2.

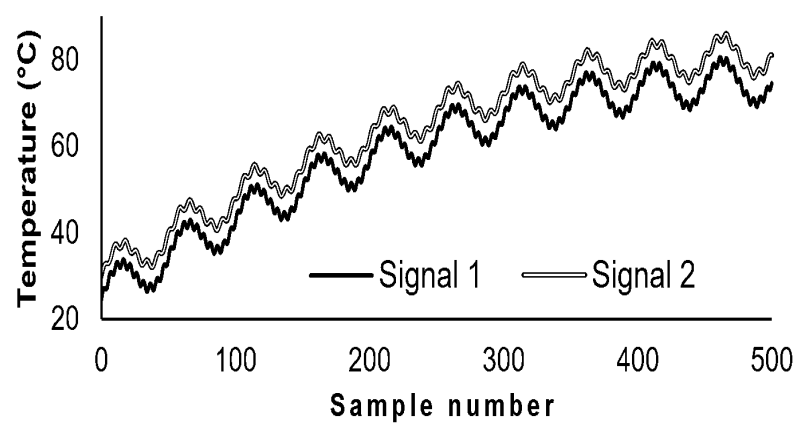

Fig. 2. The temperature signal models in graphic form.

The temperature signal filtering quality can be evaluated by phase contrast shown on (17).

$$
\text { Phase contrast }=\left|S_{P_{\text {signal } 1}}-S_{P_{\text {signal2 }}}\right|
$$

\subsection{Time shift of the correlation function}

Table II shows the contrast between the phases at different values of the correlation function time shift $\left(t_{d}\right)$.

Table 2. Contrast at different correlation function Time shift.

\begin{tabular}{|c|c|}
\hline Time shift (rad) & Phase contrast (rad) \\
\hline 0 & 0.02568011 \\
\hline-0.628 & 0.02568011 \\
\hline-1.709 & 3.115912544 \\
\hline-2.513 & 0.02568011 \\
\hline
\end{tabular}

It can be seen that the correlation function time shift does not affect the phase contrast, except when saturation occurs (the first phase is approx. 1.57, and the second phase -1.57 or vice versa). In this case, phase information is lost for the corresponding temperature signal.

\subsection{Time shift of the correlation function}

Fig. 3 shows the dependence between the contrast and percentage frequency change.

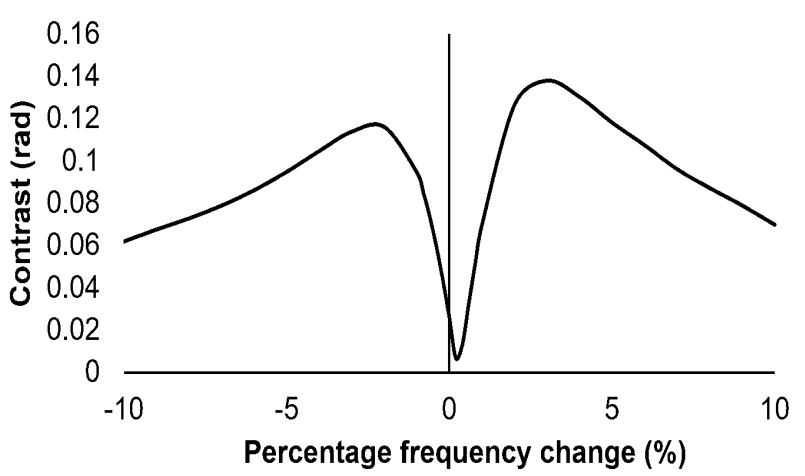

Fig. 3. The dependence between contrast and percentage frequency change.

It can be seen that the change in the correlation function frequency in narrow limits has a great effect on the phase contrast. The use of frequency change is useful in cases, where the exact lock-in frequency is not known or its value is unstable over time.

\subsection{Window length and window sliding}

In some cases, only temperature signal segment (window) can be processed. The illustrated temperature signal model consists of 500 samples (with 50 samples in one period). Fig. 4 shows the dependence between the phase contrast and window length used in postprocessing. In this case, the window lengths are multiples of the samples number in lock-in period. It should be note that the windows length can be significantly increase or decrease the phase contrast. Postprocessing over all periods can significantly reduce the value of received phase contrast.

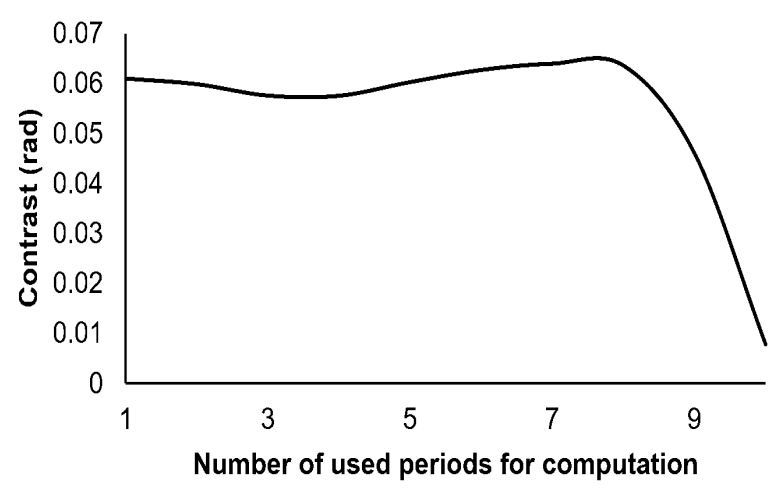

Fig. 4. The dependence between phase contrast and number of used periods in postprocessing.

Fig. 5 shows the dependence between contrast and window sliding offset at window length 350 samples (7 periods) and offset 50 samples.

It can be seen that the phase contrast tends to zero for some window sliding offset values. Therefore, the choice of the right offset is very important for filtering quality. Fig. 6 shows the same dependence as in Fig. 5, but the processing is carried out on window length, that is not multiple of the samples number in a lock-in period. 
There is only one additional sample. The computations are performed on 351 samples instead of 350 samples.

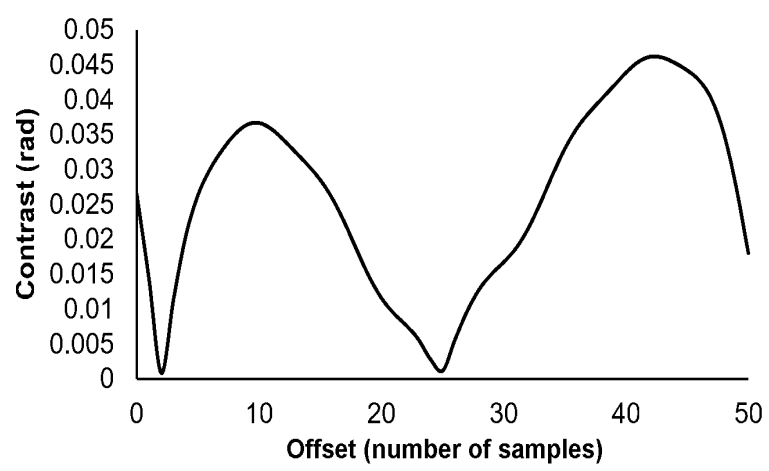

Fig. 5. The dependence between phase contrast and window length offset at using 350 samples (respectively thermograms) in processing.

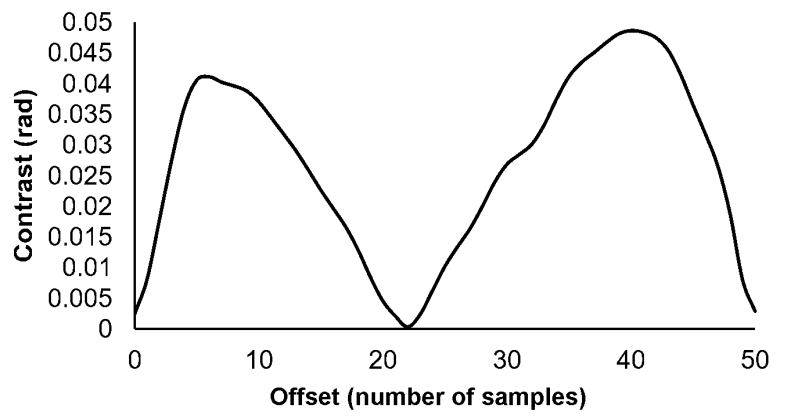

Fig. 6. The dependence between phase contrast and offset at using 351 samples (respectively thermograms).

The results from Fig. 6 shows that adding only one additional sample to window can significantly changing the obtained contrast - at Offset $=0$ the contrast obtained after processing of window with 350 samples is relatively high $(0.025)$, but the contrast after processing of the same window with only one additional sample is about ten times lower. Using window sliding approach an approximately the same relatively high contrast can be obtained even when the window length is not multiple of the samples number in a lock-in period (On Fig. 5 and on Fig. 6 the maximum obtained contrast is approximately the same -0.05 ).

\subsection{Discussion of the results from modelling}

The contrast of the phasegram depends from:

- $\quad$ the selected window sliding offset;

- $\quad$ the selected window length;

- the frequency of the correlation function.

The following algorithm for increasing the phase contrast, respectively temperature signal filtering quality includes next steps:

1. Selection of pixels used for phase contrast evaluation - one pair of pixels or multiple pairs of pixels can be selected (it is recommended pair of pixels to be chosen from areas with and without non-homogeneities, respectively);
2. Selection of window length - processing at different window length and comparison of the obtained phase contrast;

3. Window slide offset selection - changes the offset value from 1 to the number of thermograms in one period and comparison of the obtained phase contrast;

4. Choice of percentage frequency change performing multiple calculations at different percentage frequency change and comparison of the obtained phase contrast.

If saturation occurs at steps 2,3 or 4 (contrast close to 3.14), the corresponding step can be repeated at different correlation function initial phase values until a saturation disappears.

\section{Experimental work and Discussion}

One-layer printed circuit board (PCB) was performed to demonstrate experimentally the described dependencies. The side of the PCB without copper paths is heated with a halogen lamp. The light intensity of the halogen lamp is modulated with a sinusoidal modulation signal. The same side of the PCB is captured with infrared camera (reflection mode lock-in thermography). Measurement parameters are: lock-in frequency - $0.2 \mathrm{~Hz}$ (lock-in period $-1 / 0.2=5 \mathrm{~s}$ ); Number of lock-in periods - 10; frame rate of the camera - 10 frames per second (therefore, there are 50 thermograms in one lock-in period $-5 \mathrm{~s} \times 10$ frames per second).

\subsection{Saturation in phasegram}

Fig. 7 shows phasegram at Delay $=0 \mathrm{~s}$ (a) and at Delay $=500 \mathrm{~ms}(\mathrm{~b})$.

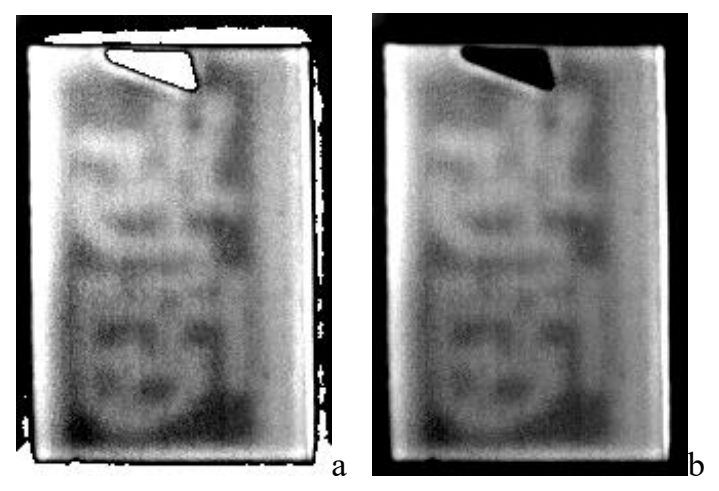

Fig. 7. Phasegram at Delay $=0 \mathrm{~s}$ (a) and at Delay $=500 \mathrm{~ms}(\mathrm{~b})$.

It can be seen that in Fig. $7 \mathrm{~b}$ there is no saturation while the contrast is the same, which is a confirmation of the results shown in Table II.

\subsection{Selection of window length}

Calculations are performed at window length 150, 350, and 500 samples (respectively 3, 7, and 10 lock-in periods). Table 3 shows the resulting contrast values. 
Table 3. Contrast at different number of lock-in periods used for computation.

\begin{tabular}{|c|c|}
\hline Number of lock-in periods & Contrast (rad) \\
\hline 3 & 0.016186 \\
\hline 7 & 0.015644 \\
\hline 10 & 0.014452 \\
\hline
\end{tabular}

It can be seen that, where it is used all lock-in periods for computation the contrast can be lower. This result confirms the dependence obtained after modelling, shown on Fig. 5.

\subsection{Performing window sliding approach}

Fig. 8 shows phasegrams obtained after computation using same window length (150), but the window sliding offset is different.

The contrast of the phasegrams shown in Table IV is significantly different after postprocessing at same window length, but different window sliding offset. This result confirms the dependence obtained in Fig. 5.
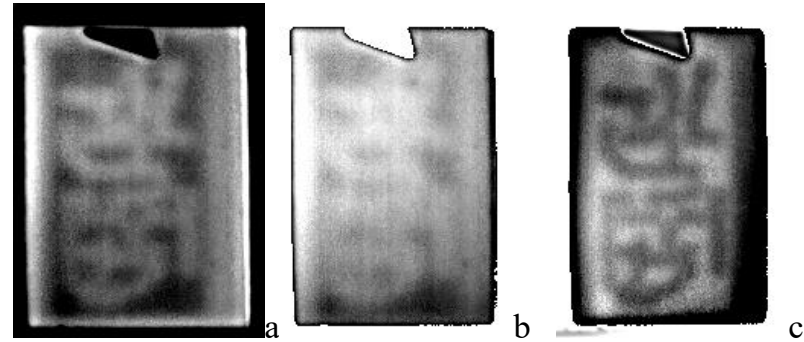

Fig. 8. Phasegram at used different part of thermogram sequence (different window sliding offset) $(\mathrm{a}-301-450, \mathrm{~b}-$ 309-458, c - 328-477).

Table 4. Contrast at different window sliding offset.

\begin{tabular}{|c|c|}
\hline Used thermograms & Contrast (rad) \\
\hline $301-450$ & 0.015952 \\
\hline $309-458$ & 0.00079604 \\
\hline $328-477$ & 0.12701 \\
\hline
\end{tabular}

Fig. 9 shows phasegrams obtained after computation at window length not multiple of the samples number in a lock-in period.
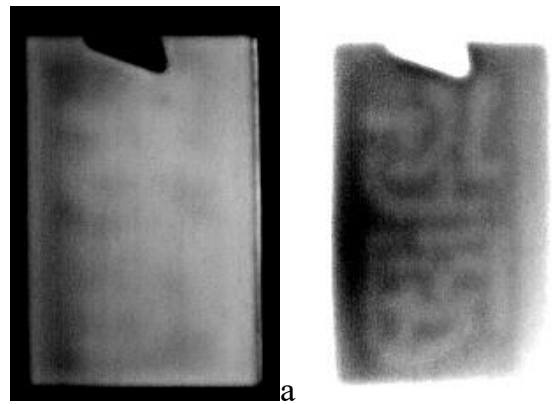

$\mathrm{b}$

Fig. 9. Phasegram obtained after computation using on window length, that is not multiple of the samples number in a lock-in period $(\mathrm{a}-300-450, \mathrm{~b}-332-482)$.

Received result confirms the dependence obtained in Fig. 6.

\subsection{Selection of percentage frequency change}

It is performed percentage frequency change calculation for computation with obtained maximum contrast (328477 ). The range of percentage frequency change (d) is $\pm 0.1 \%$ and the step is $0.001 \%$. The maximum value of the contrast is obtained at $\mathrm{d}=0.013 \%$. The contrast increases slightly $(0.12847)$.

\section{Conclusions}

The presented results shows that by using of the proposed methods can be avoided decreasing of temperature signal filtering quality due to improper postprocessing. In addition, the proposed methods allow same defect detectability at lower energy, induced in tested sample, which is very useful for materials that are not sufficiently heat-resistant.

In future work, the proposed algorithm will be applied in postprocessing after measurement of different samples with artificial defects in order to determine the effect in searching various defect types. Also will be studied possibilities for increasing the speed of postprocessing.

\section{Acknowledgment}

The paper is published with the support of the project No. DN 17/16 of the National Science Fund.

\section{References}

1. M. Vollmer, K.-P. Möllmann, Infrared Thermal Imaging: Fundamentals, Research and Applications, (Wiley-VCH, 2018)

2. C. Antolis, N. Rajic, Procedia Engineering, 188 (2017), pp. 471-478

3. O. Breitenstein, W. Warta, M. Langenkamp, Lock-in Thermography: Basics and Use for Evaluating Electronic Devices and Materials, (Springer, New York, 2010)

4. G. Pitarresi, Experimental Mechanics, 55, 4 (2015), pp. $667-680$

5. A. Stoynova, B. Bonev, MATEC Web of conferences, 125 (2017), article number 05016, Oct.2017 